\title{
Boundary-layer transition on broad cones rotating in an imposed axial flow
}

\author{
S. J. Garrett* \\ Department of Mathematics, University of Leicester, UK \\ Z. Hussain and S. O. Stephen \\ School of Mathematics, University of Birmingham, UK
}

\begin{abstract}
We present stability analyses for the boundary-layer flow over broad cones (half-angle $\psi>40^{\circ}$ ) rotating in imposed axial flows. Preliminary convective instability analyses are presented that are based on the Orr-Sommerfeld equation for a variety of axial-flow speeds. The results are discussed in terms of the limited existing experimental data and previous stability analyses on related bodies. The results of an absolute instability analysis are also presented which are intended to further those by Garrett \& Peake ${ }^{21}$ through the use of a more rigorous steady-flow formulation. Axial flow is seen to delay the onset of both convective and absolute instabilities.
\end{abstract}

\section{Introduction}

The rotating-disk boundary layer has long been used as a model for swept-wing flow because of the similarity between the basic-flow velocity profiles of the disk and the swept wing. ${ }^{1-3}$ For this reason research into the stability of rotating 3D boundary-layer flows has been focused on the disk; very little theoretical work had been published on the boundary-layer flows over rotating spheres and cones prior to 2002. However, continuing developments in spinning projectiles, aerofoils, aeroengines and other industrial applications has led to the need to understand the onset of laminar-turbulent transition of the boundary-layer flows over rotating disks, spheres and cones as objects in their own right.

For example, rotating spheres and cones are used as nose cones in aeroengine and spinning projectile applications. Here laminar-turbulent transition within the boundary-layer flow over the nose cones can lead to significant increases in drag. For aeroengine applications this has negative implications for the fuel efficiency through increased noise and energy dissipation, and for projectile applications this has negative implications for control and accurate targeting. Understanding the stability of such boundary-layer flows and developing strategies to maintain laminar flow will lead to modifications in the design of these applications and enable significant cost savings. Furthermore, flows arising from rotating disks are present in types of chemical vapour deposition (CVD) reactors used for depositing thin films of optical and electrical materials on substrates in the electrochemical industry. Such reactors operate by forcing a carrier gas containing the reactive molecules onto the substrate held within a disk-like support placed horizontally in the flow. The gas flow can be considered as a uniform axial flow incident on a rotating disk and it is desirable that the flow close to the substrate be laminar and free from instability to ensure uniform deposition. Although a large amount of literature exists on the theoretical and experimental study of the reactor-flow parameters, ${ }^{4-6}$ these are concerned with the changes in the laminar-flow profiles that can be achieved and the affect these have on deposition growth rates.

Although numerous flow-visualization studies due to Kobayashi et $a l^{7-17}$ and recent theoretical studies due to Garrett \& Peake ${ }^{18-22}$ have been published on the boundary-layer flows over rotating spheres and cones, a complete understanding of the stability characteristics of such boundary layers with regards to these applications is still a long way off. This current paper is part of a series by the present authors ${ }^{23,24}$ which considers the convective instability of the boundary-layer flow over a family of rotating cones (including the

*Presenting author. Email: s.garrett@mcs.le.ac.uk, tel: +44 (0)116 2523899. 
disk as the limiting half angle), both in and out of an imposed axial flow. Particular emphasis is placed on the above applications. The series presents complementing numerical and asymptotic studies and commenced with an investigation into a family of cones rotating in an otherwise still fluid. ${ }^{23}$ This was then followed by an investigation into the disk rotating in an imposed axial flow. ${ }^{24}$ As we shall see in $\S I I . A$, the mathematical formulation of the rotating-disk problem in axial flow is necessarily different to that for the rotating cone in axial flow, and this motivates separate publication. This paper is different in structure to the previous two investigations in that it presents preliminary numerical results only; the asymptotic analysis is still in progress and will be published separately at a later date. In addition, this paper includes an investigation into the absolute instability of the boundary-layer flow, which is associated with the onset of turbulence. ${ }^{18-22,25,26}$

Experimental observations of the transition over rotating cones have noted a distinction between the transition region on slender and broad cones. For example, experimental studies ${ }^{8}$ of cones with slender half-angles rotating in still fluid show the existence of pairs of counter-rotating Görtler-type vortices. These arise from a dynamic instability induced by the centrifugal force of the flow field. However, as the halfangle is increased beyond $\psi=30^{\circ}$, experimental results clearly show that the vortices change from pairs of counter-rotating vortices to co-rotating crossflow vortices as observed on rotating disks and spheres. The observed instability for slender cones therefore stems from an inherently different process. Indeed, Garrett et $a l .{ }^{23}$ hypothesize the existence of a viscous-mode dominated structure for slender cones $\left(\psi \leq 40^{\circ}\right)$ which leads to the onset of a centrifugal Görtler instability that is more dangerous than the usual type I and type II modes.

Further evidence for different stability characteristics of slender and broad rotating cones is obtained from the recent experimental measurements for the onset of turbulence in otherwise still fluid by Nickels (personal communication, 2007). The theoretical prediction for the onset of absolute instability due to Garrett \& Peake ${ }^{21}$ is independent of half-angle and occurs at local Reynolds number $R \approx 2.5 \times 10^{5}$. Figure 1 shows Nickels' results and we see that the onset of turbulence for the non-slender cone $\left(\psi=60^{\circ}\right)$ is in good agreement with the predicted onset of absolute instability and is independent of rotation rate. However, the onset of turbulence for slender cones $\left(\psi=30^{\circ} \& 15^{\circ}\right)$ is well in advance of the predicted onset of absolute instability and dependent on the rotation rate. This observation clearly demonstrates that transition on slender cones is inconsistent with the onset of absolute instability. Indeed, Nickels notes different behaviour in the turbulent intensity through transition in the case of the most slender cone which suggests a significantly different transition mechanism.

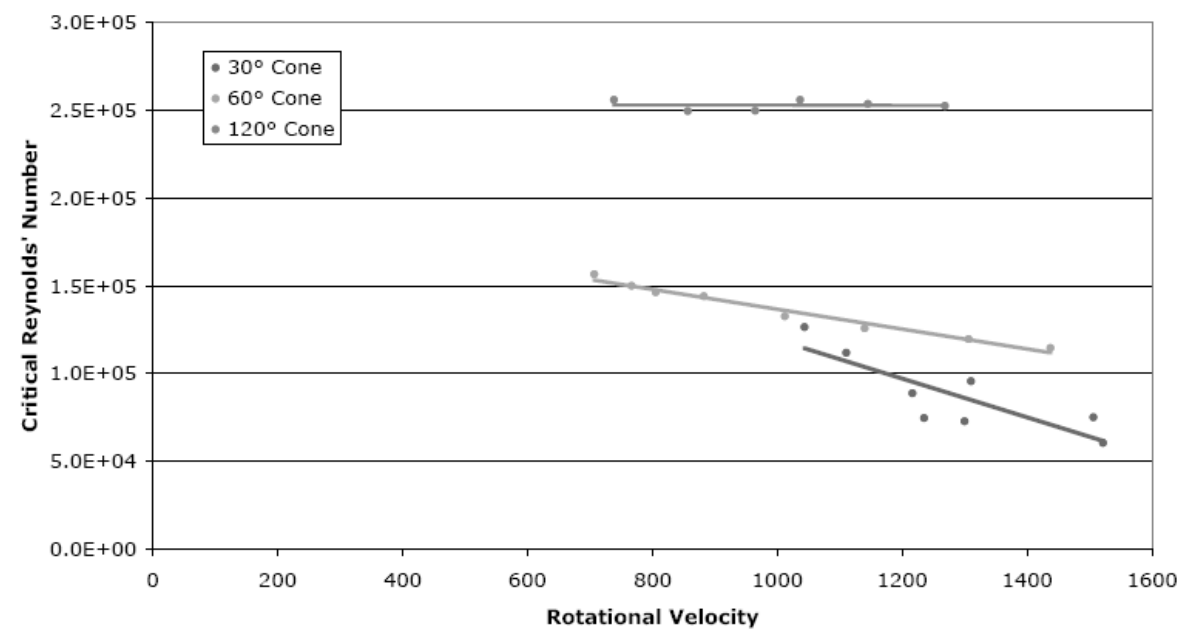

Figure 1. Experimental data due to Nickels for the onset of turbulence on rotating cones, uppermost plot is $\psi=60^{\circ}$. (Cone angle $=2 \psi$ )

This paper focuses on the impact of axial flow on the stability of the boundary layers over broad rotating cones $\left(\psi>40^{\circ}\right)$. An investigation into the hypothesized Görtler modes is underway and will be published at a later date.

With regards to similar theoretical studies, Kobayashi et al. ${ }^{15,16}$ use local-linear stability theory to predict the onset of spiral vortices in the boundary-layer flows over cones rotating in a uniform axial flow. 
Both papers consider a cone with a half-angle of $15^{\circ}$ and predict the critical Reynolds number for the onset of the vortices, the vortex angle and the number of vortices as a function of an axial-flow parameter. The predictions are found to be qualitatively consistent with experimental measurements. ${ }^{16,17}$ However, it is now clear that the transition mechanisms for slender cones are different for broad cones, which would explain why quantitative agreement was not obtained using their formulation.

Section IV of this paper is related to Garrett \& Peake's ${ }^{21}$ work on the absolute instability of the rotatingcone boundary layer in axial flow. The formulation of the laminar-flow profiles presented in this paper have been considerably improved and now lead to more physically relevant flows. The absolute instability results contained here should therefore be considered as replacing those due to Garrett \& Peake, although their results for the cone rotating in an otherwise still fluid remain unchanged.

\section{Formulation}

\section{II.A. Basic flow}

Consider a rigid cone of circular cross-section and half-angle $\psi$ that is rotating about its axis of symmetry with angular velocity $\Omega^{\star}$. Note that an asterisk denotes a dimensional quantity in all that follows. The cone is placed in a fluid with an oncoming axial flow parallel to its axis of rotation. We choose the fixed orthogonal curvilinear coordinate system $\left(x^{\star}, \theta, z^{\star}\right)$ representing streamwise, azimuthal and surface-normal variation respectively, with the origin located at the apex of the cone. A diagram can be found in Figure 1 of Garrett \& Peake. ${ }^{21}$ The local cross-sectional radius of the cone is $r_{\circ}^{\star}=x^{\star} \sin \psi$.

An appropriate coordinate transformation leads to the full Navier-Stokes equations in the coordinate system

$$
\begin{gathered}
\frac{\partial U^{\star}}{\partial x^{\star}}+\frac{U^{\star} \sin \psi}{h^{\star}}+\frac{1}{h^{\star}} \frac{\partial V^{\star}}{\partial \theta}+\frac{\partial W^{\star}}{\partial z^{\star}}+\frac{W^{\star} \cos \psi}{h^{\star}}=0, \\
\frac{\partial U^{\star}}{\partial t^{\star}}+U^{\star} \frac{\partial U^{\star}}{\partial x^{\star}}+\frac{V^{\star}}{h^{\star}} \frac{\partial U^{\star}}{\partial \theta}+W^{\star} \frac{\partial U^{\star}}{\partial z^{\star}}-\frac{V^{\star 2} \sin \psi}{h^{\star}}= \\
-\frac{1}{\rho^{\star}} \frac{\partial P^{\star}}{\partial x^{\star}}+\nu^{\star}\left(\nabla^{2} U^{\star}-\frac{2 \sin \psi}{h^{\star 2}} \frac{\partial V^{\star}}{\partial \theta}-\frac{\sin \psi}{h^{\star 2}}\left(U^{\star} \sin \psi+W^{\star} \cos \psi\right)\right), \\
\frac{\partial V^{\star}}{\partial t^{\star}}+U^{\star} \frac{\partial V^{\star}}{\partial x^{\star}}+\frac{V^{\star}}{h^{\star}} \frac{\partial V^{\star}}{\partial \theta}+W^{\star} \frac{\partial V^{\star}}{\partial z^{\star}}+\frac{V^{\star}}{h^{\star}}\left(U^{\star} \sin \psi+W^{\star} \cos \psi\right)= \\
\frac{\partial W^{\star}}{\partial t^{\star}}+U^{\star} \frac{\partial W^{\star}}{\partial x^{\star}}+\frac{V^{\star}}{h^{\star}} \frac{\partial W^{\star}}{\partial \theta}+V^{\star} \frac{\partial W^{\star}}{\partial z^{\star}}-\frac{V^{\star 2} \cos \psi}{h^{\star}}= \\
-\frac{1}{\rho^{\star}} \frac{\partial P^{\star}}{\partial z^{\star}}+\nu^{\star}\left(\nabla^{2} W^{\star}-\frac{2 \cos \psi}{h^{\star 2}} \frac{\partial V^{\star}}{\partial \theta}-\frac{\cos \psi}{h^{\star 2}}\left(U^{\star} \sin \psi+W^{\star} \cos \psi\right)\right), \\
\left.h^{\star 2}\right),
\end{gathered}
$$

where

$$
\nabla^{\star 2}=\frac{\partial^{2}}{\partial x^{\star 2}}+\frac{1}{h^{\star 2}} \frac{\partial^{2}}{\partial \theta^{\star}}+\frac{\partial}{\partial z^{\star 2}}+\frac{\sin \psi}{h^{\star}} \frac{\partial}{\partial x^{\star}}+\frac{\cos \psi}{h^{\star}} \frac{\partial}{\partial z^{\star}},
$$

is the Lapacian operator and $h^{\star}=x^{\star} \sin \psi+z^{\star} \cos \psi$. Note that $\mathbf{U}=\left(U^{\star}, V^{\star}, W^{\star}\right)$ is the velocity flow field and $P^{\star}$ the fluid pressure.

At the edge of the boundary layer the dimensional surface velocity distribution (i.e. the slip velocity) along the cone, $U_{\circ}^{\star}\left(x^{\star}\right)$, is given by the well-known potential-flow solution discussed by, for instance, $\operatorname{Rosenhead}^{27}$ and Evans. ${ }^{28}$ It takes the power-law form

$$
U_{\circ}^{\star}\left(x^{\star}\right)=C^{\star} x^{\star m}
$$

The value of the parameter $m$ is related to the cone half-angle (for instance, $m=1$ when $\psi=90^{\circ}$ and $m=0.1$ when $\psi=27.72^{\circ}$ ), and $C^{\star}$ is a scale factor determined by the free-stream axial flow incident on the 
rotating cone. This inviscid solution is a good representation of the real slip velocity, since the separated boundary layer from the cone will tend to run parallel to the cone surface.

The appropriate boundary conditions are given by the slip and no-slip conditions at the edge of the boundary layer and the cone surface, respectively. These are represented as

$$
\begin{aligned}
& U^{\star}=0, \quad V^{\star}=x^{\star} \Omega^{\star} \sin \psi, \quad W^{\star}=0 \quad \text { on } \quad z^{\star}=0, \\
& U^{\star} \rightarrow U_{\circ}^{\star}\left(x^{\star}\right), \quad V^{\star} \rightarrow 0 \quad \text { as } \quad z^{\star} \rightarrow \infty .
\end{aligned}
$$

The local axial-flow parameter $T_{s}$ is defined as

$$
T_{s}=\frac{C^{\star} x^{\star m}}{x^{\star} \Omega^{\star} \sin \psi},
$$

and is the ratio of the local slip velocity at $x^{\star}$ to the rotational speed of the cone surface at that location. It is important to note that in the case of $m \neq 1$ (i.e. $\psi \neq 90^{\circ}$ ), $T_{s}$ is dependent on the streamwise coordinate. In effect this causes the solution originally proposed by Garrett \& Peake ${ }^{21}$ to break down, as the equations governing the mean-flow are a system of partial differential equations in terms of the normal and streamwise coordinates. For this reason we appeal to a different method of solution which is similar to that used by Koh \& Price. ${ }^{29}$ We make a similarity-type transformation yielding the boundary-layer equations in terms of the governing stream-function. This is achieved by making a Mangler transformation on the governing equations. Suppose we define a new set of streamwise and surface-normal coordinates $\bar{x}^{\star}$ and $\bar{z}^{\star}$ respectively, and corresponding velocities $\bar{U}^{\star}, \bar{V}^{\star}$ and $\bar{W}^{\star}$ given by

$$
\begin{aligned}
& \bar{x}^{\star}=\frac{1}{l^{\star 2}} \int_{0}^{x^{\star}} r_{\circ}^{\star 2} d x^{\star}, \quad \bar{z}^{\star}=\frac{r_{\circ}^{\star}}{l^{\star}} z^{\star}, \\
& \bar{U}^{\star}=U^{\star}, \quad \bar{V}^{\star}=V^{\star}, \quad \bar{W}^{\star}=\frac{l^{\star}}{r_{\circ}^{\star}}\left(W^{\star}+\frac{1}{r_{\circ}^{\star}} \frac{d r_{\circ}^{\star}}{d x^{\star}} z^{\star} U^{\star}\right),
\end{aligned}
$$

where $l^{\star}$ is a typical length scale in the streamwise direction. The resulting boundary-layer equations may be derived in dimensional form using equations (1)-(4) and transformation (5), which leads to

$$
\begin{aligned}
\frac{\partial \bar{U}^{\star}}{\partial \bar{x}^{\star}}+\frac{\partial \bar{W}^{\star}}{\partial \bar{z}^{\star}} & =0, \\
\bar{U}^{\star} \frac{\partial \bar{U}^{\star}}{\partial \bar{x}^{\star}}+\bar{W}^{\star} \frac{\partial \bar{U}^{\star}}{\partial \bar{z}^{\star}}-\frac{\bar{V}^{\star 2}}{3 \bar{x}^{\star}} & =\bar{U}_{e}^{\star} \frac{\partial \bar{U}_{e}^{\star}}{\partial \bar{x}^{\star}}+\nu^{\star} \frac{\partial^{2} \bar{U}^{\star}}{\partial \bar{z}^{\star 2}}, \\
\bar{U}^{\star} \frac{\partial \bar{V}^{\star}}{\partial \bar{x}^{\star}}+\bar{W}^{\star} \frac{\partial \bar{V}^{\star}}{\partial \bar{z}^{\star}}+\frac{\bar{U}^{\star} \bar{V}^{\star}}{3 \bar{x}^{\star}} & =\nu^{\star} \frac{\partial \bar{V}^{\star}}{\partial \bar{z}^{\star 2}},
\end{aligned}
$$

where $\bar{U}_{e}^{\star}\left(\bar{x}^{\star}\right)=U_{\circ}^{\star}\left(x^{\star}\right)=C^{\star} x^{\star m}$. Applying the same transformations to the boundary conditions leads to

$$
\begin{aligned}
& \bar{U}^{\star}=0, \quad \bar{V}^{\star}=\bar{V}_{w}=\omega^{\star} \bar{x}^{\star 1 / 3}, \quad \bar{W}^{\star}=0 \quad \text { on } \quad \bar{z}^{\star}=0, \\
& \bar{U}^{\star} \rightarrow \bar{U}_{e}^{\star}=\bar{C}^{\star} \bar{x}^{\star m / 3}, \quad \bar{V}^{\star} \rightarrow 0 \quad \text { as } \quad \bar{z}^{\star} \rightarrow \infty,
\end{aligned}
$$

where

$$
\bar{C}^{\star}=C^{\star}\left(\frac{3 l^{\star 2}}{\sin ^{2} \psi}\right)^{m / 3} \text { and } \omega^{\star}=\Omega\left(3 l^{\star 2} \sin \psi\right)^{1 / 3} .
$$

We then use the following similarity-type transformation which involves the stream-function in the form

$$
\bar{\psi}=\left(\frac{6 \nu^{\star} \bar{x}^{\star} \bar{U}_{e}^{\star}}{m+3}\right)^{1 / 2} f\left(s, \eta_{1}\right), \quad \bar{U}^{\star} \frac{\partial \bar{\psi}}{\partial \bar{z}^{\star}}, \quad \bar{W}^{\star}=-\frac{\partial \bar{\psi}}{\partial \bar{x}^{\star}},
$$

where the parameters

$$
s=\left(\frac{\bar{V}_{w}^{\star}}{\bar{U}_{e}^{\star}}\right)^{2}=\left(\frac{\omega^{\star}}{\bar{C}^{\star}} \bar{x}^{\star \frac{1-m}{3}}\right)^{2} \text { and } \eta_{1}=\bar{z}^{\star}\left(\frac{m+3}{6} \frac{\bar{U}_{e}^{\star}}{\nu^{\star} \bar{x}^{\star}}\right)^{1 / 2}
$$

define the transformed coordinates for the system. The $\eta_{1}$-coordinate represents the new surface-normal coordinate scaled on displacement thickness according to the new velocity scales, whereas the $s$-coordinate 
represents the ratio of rotational flow with respect to oncoming axial flow, and is such that $s^{-1 / 2}=T_{s}$. The velocities can now be expressed as

$$
\begin{aligned}
\bar{U}^{\star} & =\bar{U}_{e}^{\star} \frac{\partial f}{\partial \eta_{1}}\left(s, \eta_{1}\right), \quad \bar{V}^{\star}=\bar{V}_{w}^{\star} g\left(s, \eta_{1}\right), \\
\bar{W}^{\star} & =-\left(\frac{6}{m+3} \nu^{\star} \bar{x}^{\star} \bar{U}_{e}^{\star}\right)^{1 / 2}\left[\left(\frac{1}{2 \bar{x}^{\star}}+\frac{1}{2 \bar{U}_{e}^{\star}}\right) f+\frac{d s}{d \bar{x}^{\star}} \frac{\partial f}{\partial s}+\frac{d \eta_{1}}{d \bar{x}^{\star}} \frac{\partial f}{\partial \eta_{1}}\right] .
\end{aligned}
$$

These, together with equations (6)-(8), lead to the governing partial differential equations that govern the laminar flow

$$
\begin{array}{r}
f^{\prime \prime \prime}+f f^{\prime \prime}+\frac{2 m}{m+3}\left(1-f^{\prime 2}\right)+\frac{2 s}{m+3}\left[g^{2}+2(1-m)\left(f^{\prime \prime} \frac{\partial f}{\partial s}-f^{\prime} \frac{\partial f^{\prime}}{\partial s}\right)\right]=0, \\
g^{\prime \prime}+f g^{\prime}-\frac{4}{m+3} f^{\prime} g+\frac{4(1-m) s}{m+3}\left(g^{\prime} \frac{\partial f}{\partial s}-f^{\prime} \frac{\partial g}{\partial s}\right)=0 .
\end{array}
$$

Note that a prime denotes differentiation with respect to $\eta_{1}$. Equations (9)-(10) are subject the nondimensional boundary conditions

$$
\begin{aligned}
& f=0, \quad f^{\prime}=0, \quad g=1 \quad \text { on } \quad \eta_{1}=0, \\
& f^{\prime} \rightarrow 1, \quad g \rightarrow 0 \quad \text { as } \quad \eta_{1} \rightarrow \infty .
\end{aligned}
$$

In order to solve the system of equations numerically, we decompose them into a fifth-order system of first-order PDEs. Solutions for $s=0$ are obtained using a fourth-order Runge-Kutta integration method with a Newton-Raphson searching routine to iterate on the boundary conditions at infinity. These initial profiles are then integrated for non-zero $s$ to obtain profiles for varying $\eta_{1}$ and $s$ using the commercial NAG routine D03DEF. The same routine has previously been used by Garrett \& Peake ${ }^{18-20}$ to obtain the basic flow over the rotating sphere both with and without an imposed axial flow.

Convective and absolute instability are local concepts and the analyses presented later will require the impulse response of the system to be determined within a parallel-flow-type approximation at a location $x^{\star}=x_{s}^{\star}$ along the surface of the cone. It is therefore appropriate to scale the steady velocities using the local-surface velocity, $x_{s}^{\star} \Omega^{\star} \sin \psi$, as

$$
U\left(\eta ; x_{s}, \psi\right)=\frac{U^{\star}}{x_{s}^{\star} \Omega^{\star} \sin \psi}, \quad V\left(\eta ; x_{s}, \psi\right)=\frac{V^{\star}}{x_{s}^{\star} \Omega^{\star} \sin \psi}, \quad W\left(\eta ; x_{s}, \psi\right)=\frac{W^{\star}}{\left(\nu^{\star} \Omega^{\star}\right)^{1 / 2}},
$$

where $\eta=z^{\star} / \delta^{\star}$ and $x_{s}=x_{s}^{\star} / \delta^{\star}$ are the non-dimensional distances scaled on the boundary-layer thickness $\delta^{\star}=\left(\nu^{\star} / \Omega^{\star}\right)^{1 / 2}$. This is consistent with the numerical stability analyses presented in the literature. ${ }^{18-21,23,24}$ Using this non-dimensionalization we note that

$$
U=s^{-1 / 2} \frac{\partial f}{\partial \eta_{1}}\left(\eta_{1}, s\right), \quad V=g\left(\eta_{1}, s\right)
$$

which are expressible in terms of the non-transformed normal coordinate $\eta$ using the following coordinate stretching for a fixed axial-flow parameter $s$

$$
\eta_{1}=\eta\left(\frac{m+3}{2 s^{1 / 2}} \sin \psi\right)^{1 / 2} .
$$

Figure 2 shows the resulting $U$ - and $V$-components of the steady flow for a variety of values of $s$. We note that these differ from those computed in Garrett \& Peake's previous absolute instability analysis, but are considered to be more accurate. We see that as $s$ increases the rotational effect dominates over the oncoming axial flow and the streamwise profiles show an inflectional nature, overshooting before converging to the inviscid solution at the edge of the boundary layer. 

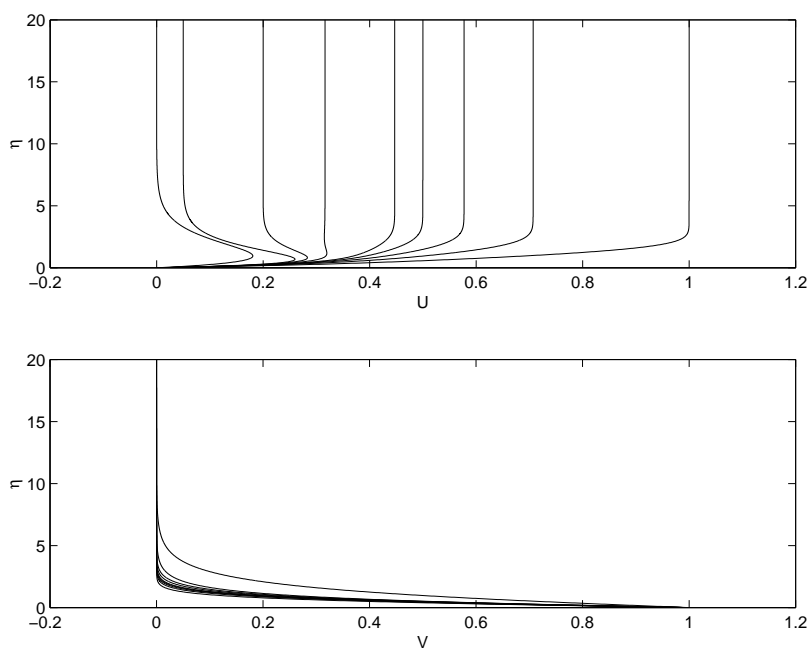

Figure 2. Basic-flow profiles for $\psi=70^{\circ}$ and $s=1,2,3,4,5,10,25,400$ and $\infty$, right to left for $U$ and bottom to top for $V$.

\section{II.B. Perturbation equations}

We use non-dimensionalizing length, velocity, pressure and time scales $\delta^{\star}, r_{\mathrm{o}, s}^{\star} \Omega^{\star}, \rho^{\star} r_{\mathrm{o}, s}^{\star 2} \Omega^{\star 2}$ and $\delta^{\star} / \Omega^{\star} r_{\mathrm{o}, s}^{\star}$ respectively. This leads to the local Reynolds number $R_{L}$ at $x_{s}^{\star}$, where

$$
R_{L}=\frac{x_{s}^{\star} \Omega^{\star} \delta^{\star} \sin \psi}{\nu^{\star}}=\frac{x_{s}^{\star} \sin \psi}{\delta^{\star}}=x_{s} \sin \psi=r_{\circ, s} .
$$

The governing disturbance equations at location $r_{\circ, s}$ are formed by perturbing the mean flow with quantities

$$
(\hat{u}, \hat{v}, \hat{w}, \hat{p})=(u(\eta), v(\eta), w(\eta), p(\eta)) \exp \left(\mathrm{i}\left(\alpha x+\beta R_{L} \theta-\gamma t\right)\right) .
$$

Physically, $\alpha$ is the disturbance wavenumber in the streamwise direction, $\beta$ is the wavenumber in the azimuthal direction and $\gamma$ is the disturbance frequency. The quantities $\alpha$ and $\gamma$ are in general complex, as required by the spatio-temporal analysis presented later. In contrast, in order to enforce periodicity round the cone, $n=\beta R_{L}$ (identified as the number of vortices) must be interpreted at real integer values only. Note that these perturbing quantities are slightly different to those used by Garrett \& Peake through the removal of a factor of $\sin \psi$ multiplying $\alpha$. This is a minor adjustment and makes the formulation consistent with the more recent studies ${ }^{23,24}$ by the present authors.

Substitution of the perturbing quantities into the general form of the Navier-Stokes equations (1)-(4) and non-dimensionalizing leads to the linear perturbation equations. These may be written as a set of six first-order equations using the transformed variables ${ }^{\mathrm{a}}$ :

$$
\begin{aligned}
& \phi_{1}=\bar{\alpha} u+\beta v, \quad \phi_{2}=\bar{\alpha} D u+\beta D v, \quad \phi_{3}=w, \\
& \phi_{4}=p, \quad \phi_{5}=\bar{\alpha} v-\beta u, \quad \phi_{6}=\bar{\alpha} D v-\beta D u,
\end{aligned}
$$

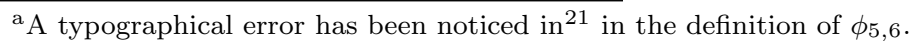


where $D$ represents differentiation with respect to $\eta$ and $\bar{\alpha}=\alpha-[i \sin \psi / R]_{s}$. These equations are

$$
\begin{aligned}
D \phi_{1}= & \phi_{2} \\
{\left[\frac{D \phi_{2}}{R_{L}}\right]_{V}=} & \frac{1}{R_{L}}\left(\left[\alpha^{2}+\beta^{2}\right]_{S}+\mathrm{i} R_{L}(\bar{\alpha} U+\beta V-\gamma)\right) \phi_{1}+\left[\frac{W \phi_{2}}{R_{L}}\right]_{S}+\left[\frac{V}{R_{L}} \beta \cos \psi+\bar{\alpha} D U+\beta D V\right] \phi_{3} \\
& +\mathrm{i}\left(\alpha \bar{\alpha}+\beta^{2}\right) \phi_{4}-\left[\frac{V \sin \psi}{R_{L}} \phi_{5}\right]_{S}+\left[\left(\bar{\alpha} \frac{\partial U}{\partial x}+\beta \frac{\partial V}{\partial x}\right) u-\frac{\sin \psi}{R_{L}}(\bar{\alpha} V-\beta U) v\right]_{S} \\
D \phi_{3}= & -\mathrm{i} \phi_{1}-\left[\frac{\phi_{3} \cos \psi}{R_{L}}\right]_{S} \\
D \phi_{4}= & {\left[\frac{\mathrm{i} W \phi_{1}}{R_{L}}\right]_{S}-\left[\frac{\mathrm{i} \phi_{2}}{R_{L}}\right]_{V}-\frac{1}{R_{L}}\left(\left[\alpha^{2}+\beta^{2}\right]_{S}+\mathrm{i} R_{L}(\alpha U+\beta V-\gamma)+D W_{S}\right) \phi_{3} } \\
D \phi_{5}= & \phi_{6}, \\
{\left[\frac{D \phi_{6}}{R_{L}}\right]_{S}=} & \frac{1}{R_{L}}\left(\left[\alpha^{2}+\beta^{2}\right]_{V}+\mathrm{i} R_{L}(\bar{\alpha} U+\beta V-\gamma)\right) \phi_{5}+\left[\frac{W \phi_{6}}{R_{L}}\right]_{S}+\left[\frac{V}{R_{L}} \bar{\alpha} \cos \psi+\bar{\alpha} D V-\beta D U\right] \phi_{3} \\
& +\left[\frac{\beta \sin \psi}{R_{L}} \phi_{4}\right]_{S}+\left[\frac{V \sin \psi}{R_{L}} \phi_{1}\right]_{S}+\left[\left(\bar{\alpha} \frac{\partial V}{\partial x}-\beta \frac{\partial U}{\partial x}\right) u+\frac{\sin \psi}{R_{L}}(\bar{\alpha} U+\beta V) v\right]_{S}
\end{aligned}
$$

where the subscripts $V$ and $S$ indicate which of the $O\left(R_{L}^{-1}\right)$ terms arise from viscous and streamlinecurvature effects, respectively. Note that the perturbation quantities $u$ and $v$ appear explicitly in (12)-(17), but can be expressed in terms of $\phi_{1}$ and $\phi_{5}$ via

$$
u=\frac{\bar{\alpha} \phi_{1}-\beta \phi_{5}}{\bar{\alpha}^{2}+\beta^{2}}, \quad v=\frac{\bar{\alpha} \phi_{5}+\beta \phi_{1}}{\bar{\alpha}^{2}+\beta^{2}} .
$$

This formulation is such that the axial-flow parameter $s$ only appears in the steady-flow equations. Equations (12)-(17) reduce to Lingwood's ${ }^{25}$ perturbation equations for the rotating disk (in an appropriate frame of reference) when $\psi=90^{\circ}$ and the basic-flow components are given by von Kármán's similarity solution. The perturbation equations differ from those stated by Garrett \& Peake ${ }^{21}$ due to the different definition of $\alpha$ and the acknowledgment that the basic-flow components are functions of both $\eta$ and $x$.

In this preliminary investigation we present a study of the Orr-Sommerfeld (OS) equation for the cone rotating in imposed axial flows. The governing fourth-order perturbation equation is obtained by neglecting streamline-curvature effects in the full perturbation equations above. This leads to

$$
\left[\mathrm{i}\left(D^{2}-k^{2}\right)^{2}+R_{L}(\alpha U+\beta V-\gamma)\left(D^{2}-\gamma^{2}\right)-R_{L}\left(\alpha D^{2} U+\beta D^{2} V\right)\right] \phi_{3}=0,
$$

where $k^{2}=\alpha^{2}+\beta^{2}$ is the effective wavenumber of the disturbance.

Analysis of the OS equation greatly simplifies the investigation as it removes the requirement for the normal velocity component $W$ and derivatives with respect to the streamwise direction. Previous studies ${ }^{18,25,31}$ have shown that although the predicted onset of instability is underestimated and the relative importance of the streamline-curvature mode (type II) is unable to be predicted, analysis of the Orr-Sommerfeld equation leads to qualitatively correct predictions which increase in numerical accuracy as $R_{L} \rightarrow \infty$. This is further discussed in $\S I I I$.

Equations (12)-(17) use a parallel-flow-type approximation in which we ignore variation in the local Reynolds number $R_{L}$ with local surface cross-sectional radius and assume that $\eta / r_{\circ}<<1$. This involves replacing the variable $r_{\circ}+\eta \cos \psi$, which appears in the coefficients of the perturbation equations, by $R_{L}$. The resulting stability results are then strictly local, with location $R_{L}=r_{\circ, s}$ appearing as a parameter. The assumption $R_{L}>>1$ (equivalent to $\delta^{\star}<<x^{\star}$ ) necessarily prohibits analysis close to the apex where $R_{L}=O(1)$. The implications of this approximation are discussed in $\S \mathrm{V}$.

\section{Convective instability}

In this section we solve the eigenvalue problem defined by the OS equation (18), with the homogeneous boundary conditions

$$
\begin{array}{ll}
\phi_{i}=0, & \eta=0, \\
\phi_{i} \rightarrow 0, & \eta \rightarrow \infty,
\end{array}
$$


where $i=1,2 \ldots 4$. This eigenvalue problem will be solved for certain combinations of values of $\alpha, \beta$ and $\gamma$ at each Reynolds number, $R_{L}$ for a particular half-angle. From these we form the dispersion relation, $D\left(\alpha, \beta, \gamma ; R_{L}, \psi\right)=0$, with the aim of studying the occurrence of convective instabilities. This allows a discussion of the spatial branches of the dispersion relation before we study their pinching in the absolute instability analysis of $\S \mathrm{IV}$. In each analysis the branches were calculated using a fourth-order Runge-Kutta integrator with Gram-Schmidt orthonormalization and a Newton-Raphson linear search procedure.

Since we are supposing here that the flow is not absolutely unstable it follows that in the Briggs-Bers procedure we can reduce the imaginary part of the frequency down to zero, so that $\gamma_{i}=0$. To produce the neutral curves for convective instability a number of approaches can be taken in this stationary frame of reference. One approach is to insist that the vortices rotate at some fixed multiple of the cone surface velocity, thereby fixing the ratio $\gamma_{r} / \beta$, and then $\alpha$ and $\beta$ are calculated using a spatial analysis. This is the approach taken here. In particular, we explicitly assume that the vortices rotate with the surface of the cone (i.e. are stationary) so that $\gamma_{i}=\beta$, which is consistent with experimental observations discussed in $\S$ I. As discussed by Corke \& Knasiak, ${ }^{30}$ traveling modes can dominate on a highly polished rotating disk and this is also likely to be the case over a highly polished broad rotating cone. However, we are particularly interested in practical engineering applications where highly polished surfaces will not be found.

\section{III.A. Cone rotating in otherwise still fluid and disk rotating in axial flow}

In publication ${ }^{23}$ the present authors give the convective instability analysis for a family of cones rotating in an otherwise still fluid. An appropriate version of the full system of perturbation equations (12)-(17) is used, and both numerical and asymptotic approaches are taken. Furthermore the equivalent analysis for a disk placed in an enforced axial flow is contained in publication. ${ }^{24}$ The interested reader is therefore referred to those publications in the limiting cases of $s \rightarrow \infty$ for all $\psi$ and $\psi=90^{\circ}$ for all $s$. We now proceed to present an analysis for non-zero axial flow incident on rotating cones (i.e. finite $s$ and $\psi \neq 90^{\circ}$.)

\section{III.B. Cone rotating in uniform axial flow}

One spatial branch was found to determine the convective instability characteristics in the OS analysis for each half-angle considered for each axial-flow rate. This branch arises from the crossflow (type I) instability mode and is identical to that discussed in related publications. ${ }^{19,20,31}$ It is known that this mode arises from the inflectional nature of the streamwise mean velocity component $U$. Previous investigations of related boundary layers have also noted the appearance of a streamline curvature (type II) instability mode. This corresponds to a centrifugal instability associated with the way in which the outer-flow streamlines are curved by an $O\left(R_{L}^{-1}\right)$ amount close to the outer edge of the boundary layer. However, since streamline-curvature effects have been removed in this preliminary investigation of the OS equation, the type II mode does not arise.

Figure 3 shows the neutral curves in the $\left(R_{L}, \alpha_{r}\right)-,\left(R_{L}, \beta\right)-,\left(R_{L}, k_{\delta}\right)$ - and $\left(R_{L}, \epsilon\right)$-planes for $\psi=70^{\circ}$ for a variety of values of $s$. Note that $k_{\delta}=\sqrt{\alpha^{2}+\beta^{2}} / \sin \psi$ is the modified wavenumber and $\epsilon=$ $\arctan \left(\beta \sin \psi / \alpha_{r}\right)$ is the modified waveangle of the disturbances. Although the introduction of the factor $\sin \psi$ appears arbitrary here, it arises from the formulation of the asymptotic study (not presented here) and enables comparison with the previous numerical and asymptotic investigations ${ }^{23,24}$ (the interested reader is referred to those publications for more details). The neutral curves are determined by setting $\gamma_{i}=0$ (i.e. a neutral disturbance) and $\gamma_{r}=\beta$ (i.e. to guarantee stationary disturbances) and for each $R_{L}$ the dispersion relation is then solved to return a pair of values $\left(\alpha_{r}, \beta\right)$ for each neutral mode. As can be seen in Figure 3 , the value of $\beta$ varies continuously, although of course only values of $\beta$ are allowed such that $n$ is integer. Inside each loop of the neutral curves the flow is convectively unstable.

Figure 3 clearly demonstrates that axial flow has a stabilizing effect on the rotating-cone boundary layer by increasing the critical Reynolds number for the onset of instability. It is also clear that the range of waveangles that the spiral vortices are predicted to exist is narrowed with increased axial flow. Table 1 lists the critical values of $R_{L}, x_{L}=R_{L} / \sin \psi$, vortex angle and number of vortices at the onset of convective instability at each axial flow rate and half-angle studied using the Orr-Sommerfeld formulation.

Unfortunately experimental results ${ }^{9,17}$ for rotating cones in axial flow exist only for $\psi=15^{\circ}$. It is therefore possible to compare our calculations for $\psi=70^{\circ}$ and $50^{\circ}$ only qualitatively with these studies. Agreement is found with the general trend that axial flow delays the onset of spiral vortices and increases their waveangle. 

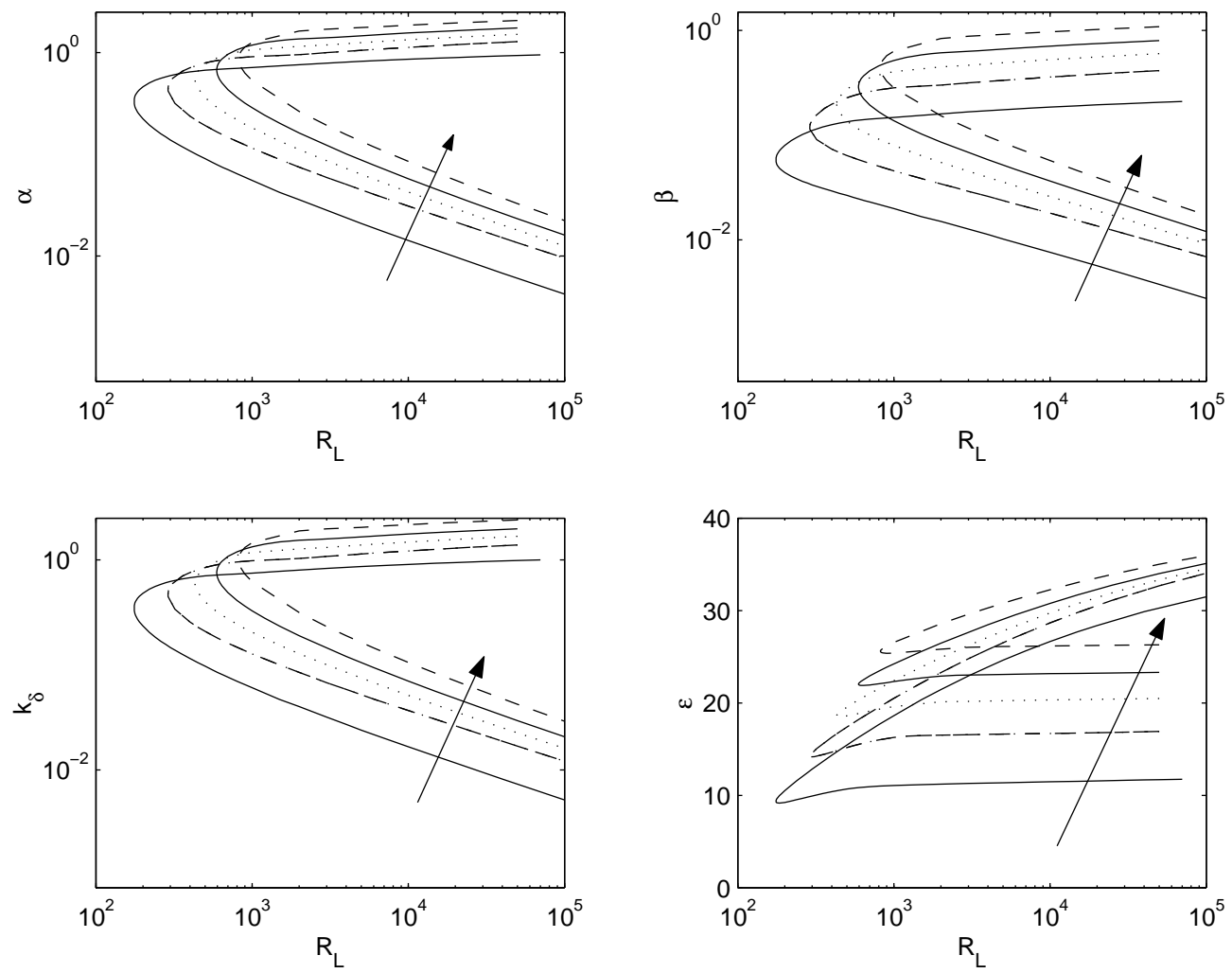

Figure 3. Orr-Sommerfeld neutral curves for convective instability of stationary vortices for $\psi=70^{\circ}$ with $s=$ $\infty, 400,25,10 \& 5$, arrow indicates direction of decreasing $s$ (i.e. increasing axial flow).

In order to compare the onset of convective instability at each $s$ between both values of $\psi$, it is sensible to use $x_{L}=R_{L} / \sin \psi$ (the non-dimensional distance along the cone surface). Table 1 shows that the onset of convective instability occurs further along the $50^{\circ}$-cone than the $70^{\circ}$-cone for each value of $T_{s}$. However, it is clear from the definition of the axial-flow parameter that equal values of $T_{s}$ for both values of $\psi$ does not translate as equal incident axial-flow rates. This is due to the appearance of $m$ which depends on $\psi$. Care must therefore be taken in making such comparisons. The interpretation of such comparisons is discussed further by Garrett ${ }^{18}$ and Garrett \& Peake. ${ }^{21}$ We are able to conclude that the critical values of the $50^{\circ}$-cone appear to more sensitive to the values of $T_{s}$ than the $70^{\circ}$-cone.

In order to discuss the relative importance of the type I and type II modes of instability it is necessary to consider the full perturbation system of equations (12)-(17), which is not done here. For the related boundary-layer flows over rotating spheres and disks, Garrett \& Peake ${ }^{20}$ and the present authors ${ }^{23}$ find that increased axial flow increases the importance of the type II (streamline curvature) mode until it becomes the most dangerous below a certain value of $s$. The suggestion that increased axial flow would emphasize the streamline-curvature mode is also sensible in this geometry. In particular, Figure 2 shows that axial flow increases the amount of fluid entrained into the boundary layer, which would clearly lead to more streamline curvature. It is also expected that this will occur on rotating cones of other half angles. Physically, it is suggested that axial flow effectively sweeps any instabilities arising from, say, surface roughness further downstream, causing the location of transition to retreat downstream also. Therefore axial flow has a stabilizing effect on convective instabilities.

Figure 4 demonstrates the implications of using the OS equation (18) instead of the full perturbation equations (12)-(17) for a cone with $\psi=70^{\circ}$ rotating in an otherwise still fluid. This is done by plotting neutral curves as in Figure 3, but calculated from each system of equations. In each plot we see a marked difference in the behaviour close to the critical value of $R_{L}$, most notably in that the two-lobed structure does not exist in the OS analysis, but also that the critical values of $R_{L}$ are lower in the OS analysis. As $R_{L}$ increases, excellent agreement is obtained in the upper-branch calculations in the $\left(R_{L}, \alpha_{r}\right)$-, $\left(R_{L}, \beta\right)$ and $\left(R_{L}, k_{\delta}\right)$-planes and good agreement is obtained in the lower branches. In the $\left(R_{L}, \epsilon\right)$-plane excellent 


\begin{tabular}{|cc|cccc|cccc|}
\hline & & \multicolumn{5}{|c|}{$\psi=70^{\circ}$} & \multicolumn{5}{|c|}{$\psi=50^{\circ}$} \\
\hline$s$ & $T_{s}$ & $R_{L}$ & $x_{L}$ & $\epsilon$ & $n$ & $R_{L}$ & $x_{L}$ & $\epsilon$ & $n$ \\
\hline$\infty$ & 0 & 175.5 & 186.8 & $9.2^{\circ}$ & $\sim 10$ & 158.5 & 206.9 & $7.6^{\circ}$ & $\sim 8$ \\
10,000 & 0.01 & 265.6 & 282.6 & $13.2^{\circ}$ & $\sim 27$ & 272.1 & 355.2 & $10.6^{\circ}$ & $\sim 29$ \\
400 & 0.050 & 289.4 & 308.0 & $14.2^{\circ}$ & $\sim 34$ & 296.2 & 386.7 & $11.5^{\circ}$ & $\sim 36$ \\
25 & 0.200 & 432.1 & 459.8 & $18.6^{\circ}$ & $\sim 85$ & 433.6 & 566.0 & $12.1^{\circ}$ & $\sim 88$ \\
10 & 0.316 & 594.1 & 632.2 & $22.0^{\circ}$ & $\sim 170$ & 588.1 & 767.7 & $18.0^{\circ}$ & $\sim 175$ \\
5 & 0.447 & 819.7 & 872.3 & $25.6^{\circ}$ & $\sim 360$ & 803.1 & 1048.4 & $21.0^{\circ}$ & $\sim 360$ \\
\hline
\end{tabular}

Table 1. Critical parameters at the onset of convective instability for a range of axial-flow rates.

agreement is obtained in the lower branch (which corresponds to the upper branch of the other plots) but the discrepancy in the predicted upper-branch waveangle is more noticeable (although this is partly to do with the log-scalings used in the other plots). The waveangle and wavenumber plots also show the comparison with the asymptotic predictions. Figure 4 therefore demonstrates that the results from this preliminary OSanalysis are expected to be quantitatively close to those arising from the full system. However, the relative importance of the type I and type II modes (as determined by the two lobed structure) cannot be investigated under the OS formulation, as mentioned above. Both the full-system numerics and the asymptotic plots in Figure 4 are due to the present authors. ${ }^{23}$ For comparison with the data in Table 1, the critical parameters at the onset of convective instability arising from the full system of equations for $\psi=70^{\circ}$ and $s=\infty$ are $R_{L} \approx 277, \epsilon=11^{\circ}$ and $n \sim 21 ;$ and for $\psi=50^{\circ}$ and $s=\infty$ are $R_{L} \approx 249, \epsilon=9^{\circ}$ and $n \sim 16$.
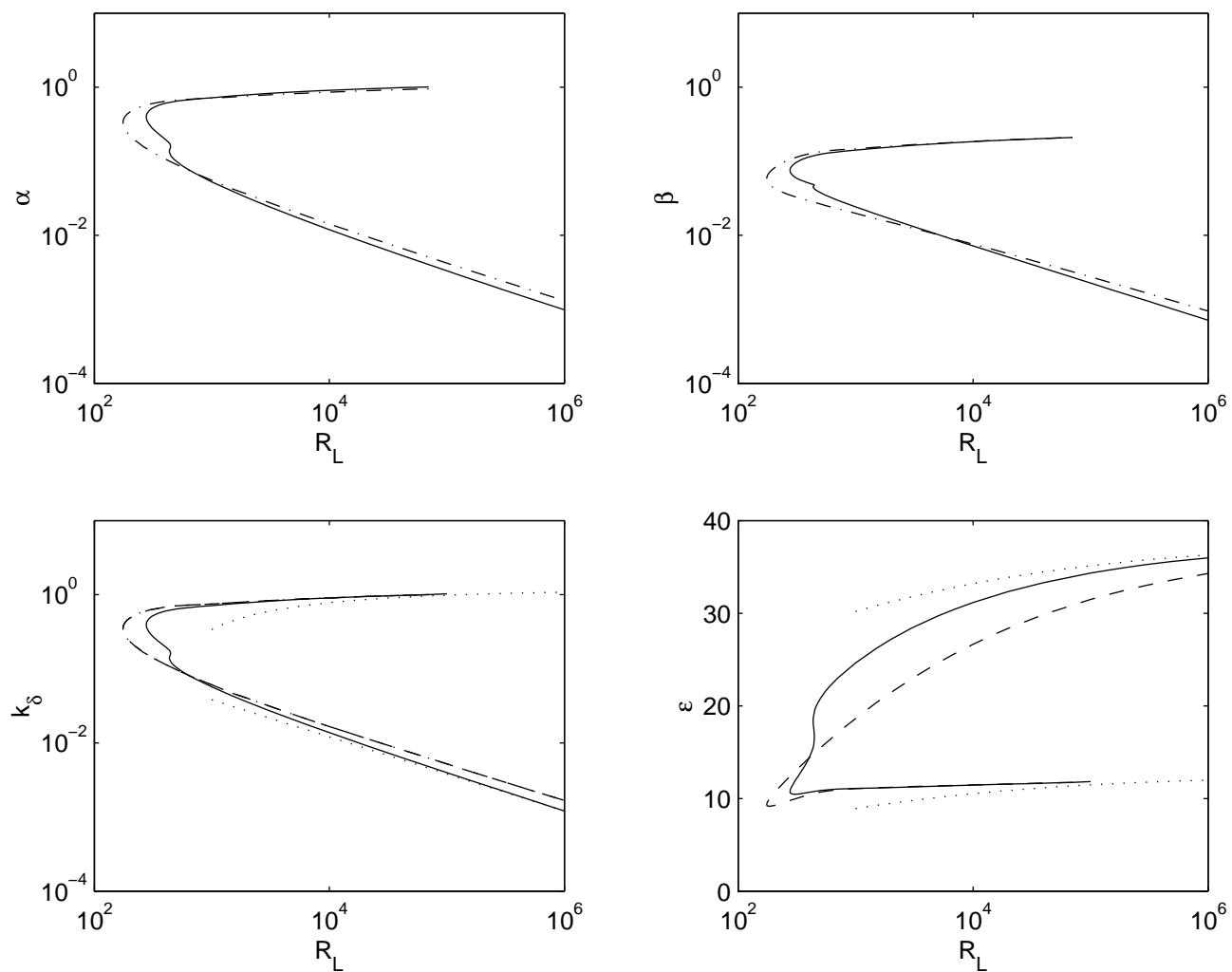

Figure 4. Comparison of Orr-Sommerfeld neutral curves (-.) with full-perturbation-system neutral curves (-) and the asymptotics $(\cdots)$ for the convective instability of stationary disturbances over a cone rotating in otherwise still fluid $(s \rightarrow \infty)\left(\psi=70^{\circ}\right)$. 


\section{Absolute instability}

We now solve the OS eigenvalue problem with the aim of studying the occurrence of absolute instability. The Briggs-Bers method is used to detect an absolutely-unstable response to the initial perturbation of the form $\delta\left(r-r_{s}\right) \delta(t) e^{\mathrm{i} \beta \theta}$. For absolute instability to occur we need to locate a pinch point $\left(\alpha^{\circ}, \gamma^{\circ}\right)$, formed by the coalescence between two spatial branches which originate from distinct half- $\alpha$-planes when $\gamma_{i}$ is sufficiently large and positive. The flow is then absolutely unstable if $\gamma_{i}>0$ at the pinch point, and is otherwise at worst convectively unstable.

A pinch point has indeed been found for each half-angle $\psi$ and flow parameter $s$, which is unstable for sufficiently large $R_{L}$. The pinch for a particular set of parameter values is shown in Figure 5 where the characteristic exchange of branches can be seen. This pinching behaviour is consistent with that previously found on related geometries. ${ }^{18-22,25,26}$ By monitoring the behaviour of the two spatial branches as the Reynolds number is varied, it is possible to determine the critical value of $R_{L}$ for the onset of absolute instability. As Lingwood ${ }^{25}$ and Garrett \& Peake ${ }^{19,20}$ have demonstrated with previous analyses of the OS/full system of perturbation equations, the pinch is formed by the coalescence of two branches which are inviscid in origin. One is the convectively unstable type I branch and the other is a convectively stable branch (typically denoted type III). Existence of a pinch in this current OS analysis would therefore demonstrate that the rotating-cone boundary layer is absolutely unstable in axial flow, although the predicted onset will be quantitatively different to that predicted by the more accurate full-perturbation system. As with the convective instability case, the extent of the discrepancy can be judged by plotting neutral curves for absolute instability for a cone rotating in otherwise still fluid. Figure 6 demonstrates the implications of using the OS equation (18) instead of the full perturbation equations (12)-(17) for a cone with $\psi=70^{\circ}$ rotating in an otherwise still fluid in terms of the $\left(R_{L}, \alpha_{r}\right)-,\left(R_{L}, \alpha_{i}\right)-,\left(R_{L}, \beta\right)$ - and $\left(R_{L}, \gamma_{r}\right)$-planes.

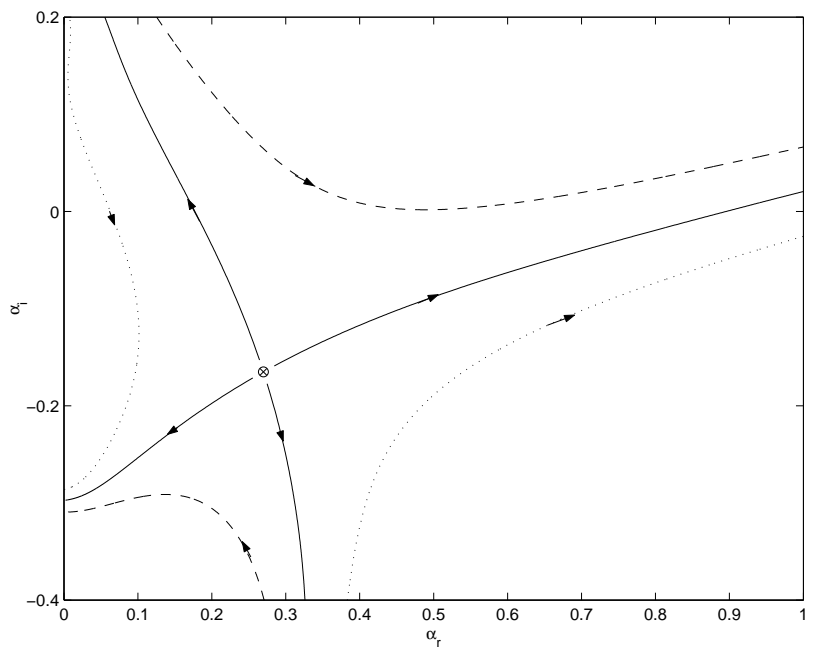

Figure 5. Branches of type 1 and type 3 for $\psi=70^{\circ}$ and $s=10,000$ for $R_{L}=3,000, \beta=0.26$ and $\gamma_{i}=0.0025$ (solid line), $\gamma_{i}=0.01$ (dashed line) \& $\gamma_{i}=-0.005$ (dotted line), arrows indicated direction of increasing $\gamma_{r}$. The pinch point is indicated by the cross.

\section{IV.A. Cone rotating in otherwise still fluid and disk rotating in axial flow}

Garrett \& Peake ${ }^{21}$ give the results of absolute-instability analyses for a family of cones rotating in a range of axial flows. They use the full system of perturbation equations. As discussed above, this current paper uses more accurate basic-flow profiles for non-zero axial flow imposed on rotating cones and so the results are intended to replace those previously published. However, the basic flows obtained using the methods detailed in §II.A are identical to those obtained previously for zero axial flow $\left(T_{s}=0, s \rightarrow \infty\right)$ for all $\psi$ and for $T_{s} \neq 0$ when $\psi=90^{\circ}$. The existing results for these cases are therefore unchanged and the interested reader is referred to Garrett \& Peake. $^{21}$ 

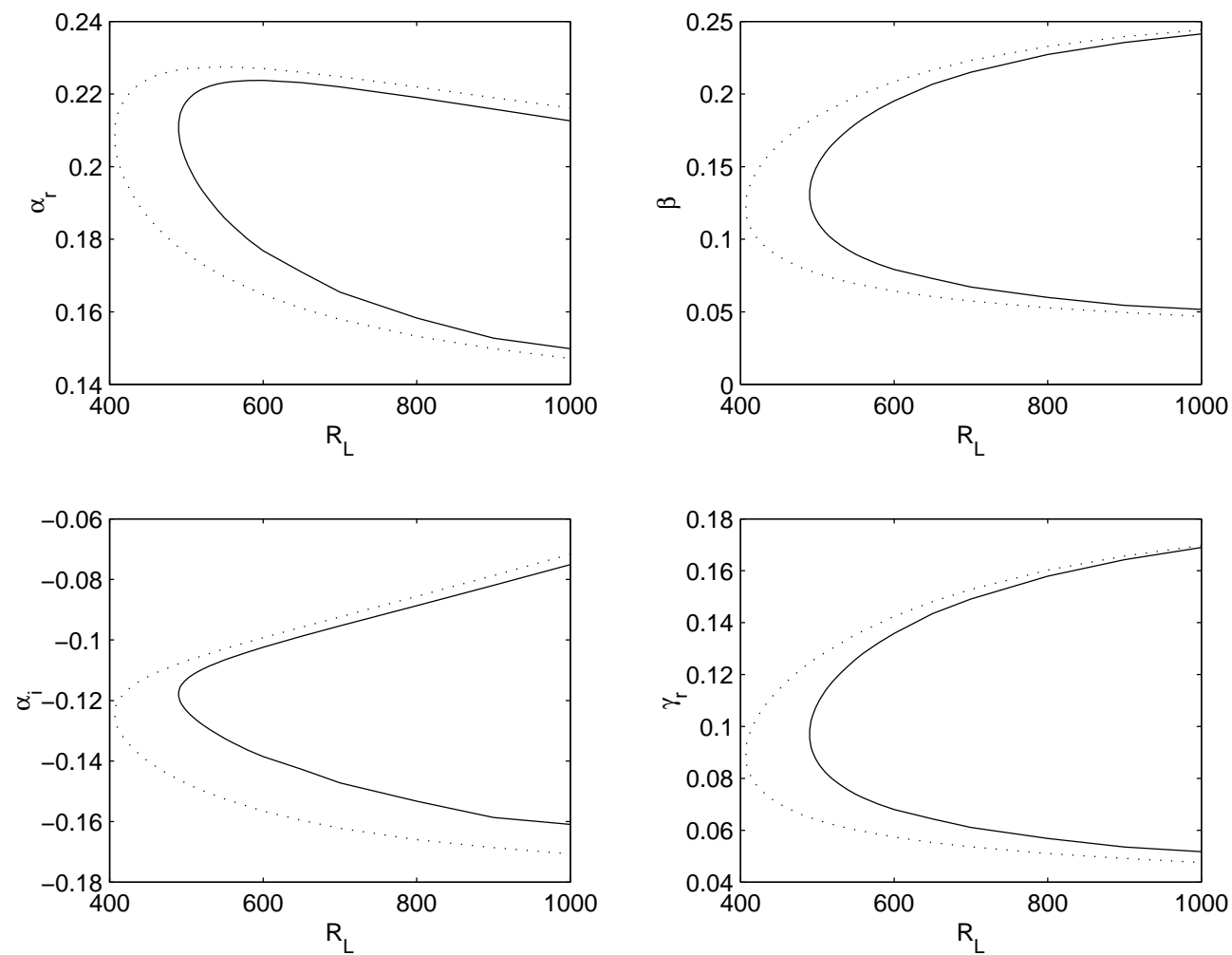

Figure 6. Comparison of Orr-Sommerfeld neutral curves ( $\cdots)$ with full-perturbation-system neutral curves (-) for absolute instability of a cone rotating in otherwise still fluid $(s \rightarrow \infty)$ for $\psi=70^{\circ}$.

\section{IV.B. Cone rotating in uniform axial flow}

Figure 7 shows neutral curves for absolute instability when $\psi=70^{\circ}$ in terms of the $\left(R_{L}, \alpha_{r}\right)-,\left(R_{L}, \alpha_{i}\right)$-, $\left(R_{L}, \beta\right)$ - and $\left(R_{L}, \gamma_{r}\right)$-planes at $s=10000,1000,400,100$ and 50 . The most striking feature of this figure is the sensitivity of absolute instability to axial flow. For example, the onset of convective instability is delayed from $R_{L} \approx 176$ to 289 as the imposed axial flow is increased from $s=\infty\left(T_{s}=0\right)$ to $s=400\left(T_{s}=0.05\right)$; this represents an increase of around $60 \%$. However, the onset of absolute instability is increased from $R_{L} \approx 407$ to 2279 over the same range of $s$; this is an increase of around $460 \%$. For this reason the figure shows the neutral curves over a much shorter range of axial-flow parameters.

Table 2 lists the critical values of $R_{L}$ and $x_{L}$ for the onset of absolute instability at each axial flow rate and half-angle studied using the Orr-Sommerfeld formulation. For comparison, the results obtained from the full perturbation system for cones rotating in zero axial-flow are $R_{L} \approx 490$ for $\psi=70^{\circ}$ and $R_{L} \approx 441$ for $\psi=50^{\circ}$. As with $\psi=70^{\circ}$, the onset of absolute instability over the $50^{\circ}$-cone is seen to be very sensitive to axial flow. As with the onset of convective instability, the delayed onset of absolute instability with $T_{s}$ for $\psi=50^{\circ}$ is more sensitive than for $\psi=70^{\circ}$.

\section{Conclusion}

In this paper we have used an Orr-Sommerfeld analysis to show that the boundary layer over a broad cone $\left(\psi>40^{\circ}\right)$ rotating in an imposed axial flow is both convectively and absolutely unstable. The onset of both instabilities are delayed with increased $T_{s}$ (i.e. decreased $s$ ) and the critical position for onset (as measured along the surface of the cone) is delayed with decreased $\psi$.

We have made use of a parallel-flow-type approximation by assuming that factors $1+\eta \cos \psi / R_{L}$ can be replaced by unity. We are careful to point out that this approximation would imply that the resulting perturbation equations are not consistent to $O\left(R_{L}^{-1}\right)$, the same order as the viscous and streamline curvature effects. Therefore, whether using the Orr-Sommerfeld equation (18) or the full perturbation equations (12)-(17), the solutions cannot be justified rigorously at finite $R_{L}$. It is the authors' opinion that this 

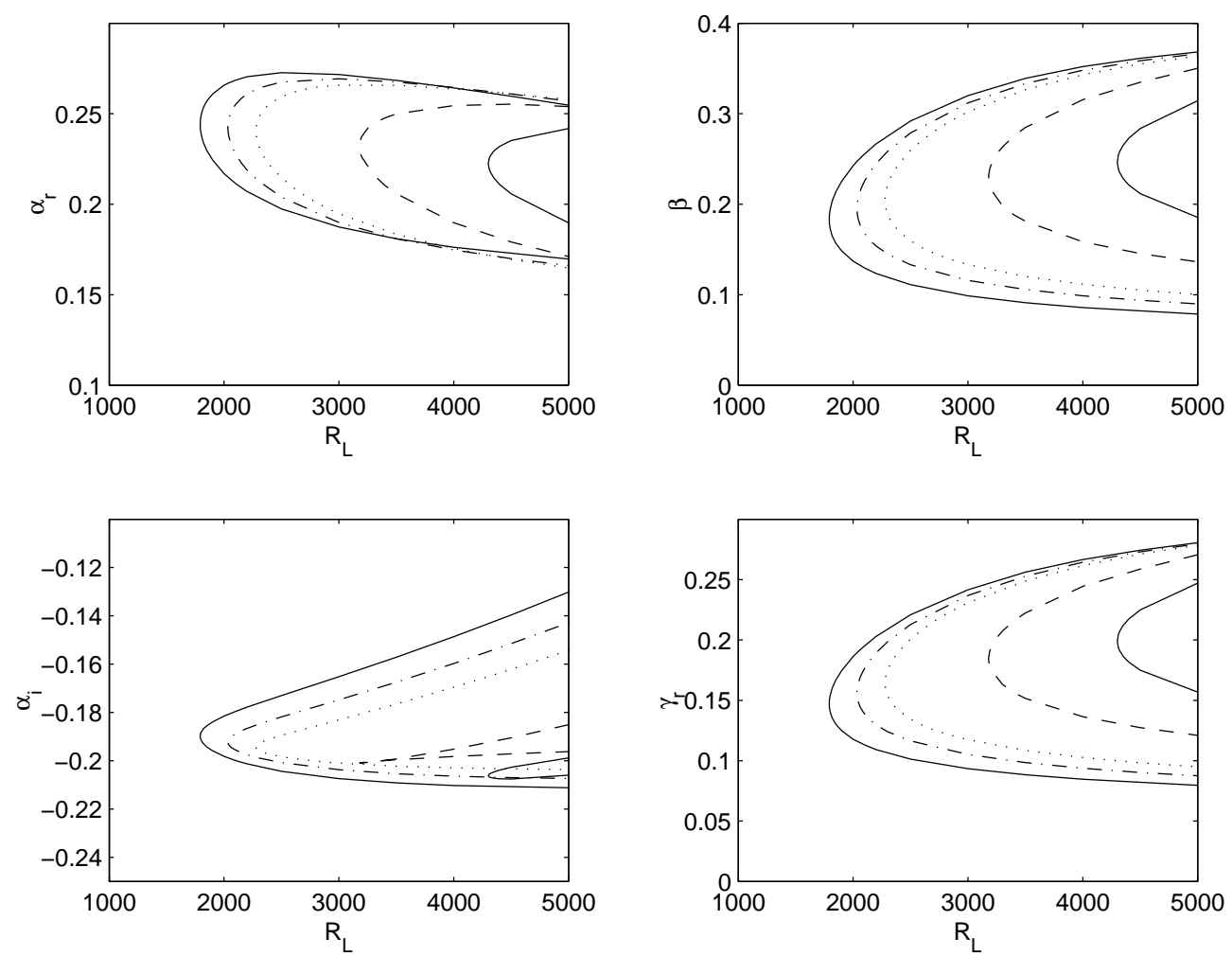

Figure 7. Orr-Sommerfeld neutral curves for absolute instability of a cone rotating in otherwise still fluid $(s \rightarrow \infty)$ for $\psi=70^{\circ}$. Curves are shown for $s=10000,1000,400,100$ and 50 from left to right (i.e. increasing axial flow).

approximation will not affect the conclusions presented here. In particular, studies into the convective instability of related boundary layers by the present authors ${ }^{23,24}$ also use this approximation and all numerical results are verified by rigorous asymptotic investigations at high $R_{L}$. Furthermore, absolute instability is known to arise from inviscid modes which are observable in the purely inviscid and rigorous analysis of the Rayleigh equation obtained by neglected both streamline curvature and viscous terms from the full system of perturbation equations. However, we do acknowledge that the results presented here will be subject to quantitative inaccuracies as a result of the approximation. A full discussion of the impact of the approximation is intended for a future paper where the complementary asymptotic study will be presented.

Unfortunately no experimental results for cones rotating in imposed axial flows are available for comparison at half-angles greater than $\psi=15^{\circ}$. This means that quantitative comparisons of both the onset of spiral vortices (against convective instability) and the onset of turbulence (against absolute instability) cannot be made. Compiling the numerical codes for $\psi=15^{\circ}$ would not be useful as this investigation is formulated to study crossflow instabilities, rather than the hypothesized Görtler modes on slender cones.

The results of this paper are restricted to local-linear stability. The question of the global-linear stability of the rotating disk was first investigated by Davies \& Carpenter, ${ }^{32}$ who use a numerical simulation to demonstrate that the global mode associated with the local absolute instability is damped. This has also been theoretically verified in the preliminary study of Garrett \& Peake ${ }^{22}$ for the boundary layers on disks and cones rotating in otherwise still fluids. It is therefore also likely to be case when axial flow is incident on the rotating cone. Note however that this does not imply that absolute instability is not involved in transition. In order to see this one must include nonlinearity: Pier et al. ${ }^{33,34}$ have shown that a self-excited, nonlinear global mode will always exist in the presence of a region of local absolute instability. This is in contrast to linear theory, since publications ${ }^{22,32}$ have demonstrated that the region of local absolute instability on the disk is not sufficient to support an unstable linear global mode. Pier ${ }^{35}$ has further shown that this nonlinear global mode can undergo secondary instability very close to the convective-absolute boundary, providing a possible route to turbulence. The global behaviour needs to be investigated for the rotating-cone boundary layer both with and without an imposed axial flow. 


\begin{tabular}{|c|c|c|c|c|c|}
\hline & & $\psi=70^{\circ}$ & & $\psi=50^{\circ}$ & \\
\hline$s$ & $T_{s}$ & $R_{L}$ & $x_{L}$ & $R_{L}$ & $x_{L}$ \\
\hline$\infty$ & 0 & 406.4 & 432.5 & 368.0 & 480.4 \\
\hline 10000 & 0.010 & 1792.9 & 1908.0 & 1669.4 & 2179.2 \\
\hline 1000 & 0.032 & 2033.9 & 2164.4 & 1891.2 & 2468.8 \\
\hline 400 & 0.050 & 2278.8 & 2425.0 & 2116.2 & 2762.5 \\
\hline 100 & 0.100 & 3180.5 & 3384.6 & 2947.3 & 3847.4 \\
\hline 50 & 0.141 & 4301.0 & 4577.0 & 3982.3 & 5198.5 \\
\hline 25 & 0.200 & 6882.5 & 7324.2 & 6372.4 & 8318.6 \\
\hline
\end{tabular}

Table 2. Critical parameters at the onset of absolute instability for a range of axial-flow rates.

In all papers in this series an incompressible boundary layer has been assumed and methods from linearstability theory applied. Although the assumption of incompressibility is reasonable for many aeroengine applications, it is not for the high-speed aerodynamic and CVD applications discussed in $\S$ I. The investigations performed so far, although important in a theoretical context, must be considered as preliminary investigations with regards to such applications. Further work is therefore intended to study the effects of compressibility on the instability modes discussed. Work is also underway to clarify the transition mechanisms on slender cones.

\section{Acknowledgments}

SJG wishes to acknowledge study leave granted by the University of Leicester. ZH wishes to acknowledge financial support from the School of Mathematics, University of Birmingham and the EPSRC.

\section{References}

${ }^{1}$ Reed, H. L. \& SARIC, W. S. 1989 'Stability of three-dimensional boundary layers.' Ann. Rev. Fluid Mech. 21, 235-84.

${ }^{2}$ Reshotko, E. 1994 'Boundary-layer instability, transition and control.' AIAA J. 94, 0001.

${ }^{3}$ Saric, W. S., Reed, H. L. \& White, E. B. 2003 'Stability and transition of three-dimensional boundary layers.' Ann. Rev. Fluid Mech. 35, 413-40.

${ }^{4}$ Chen, K. \& Mortazavi, A. R. 1986 'An analytic study of the chemical vapor deposition (CVD) processes in a rotating pedestal reactor.' J. Crystal Growth 77, 199-208.

${ }^{5}$ Coltrin, M. E., Kee, R. J. \& Evans, G. H. 1989 'A mathematical model of the fluid mechanics and gas-phase chemistry in a rotating disk chemical vapor deposition reactor.' J. Electrochem Soc. 136, 819-829.

${ }^{6}$ Vanka, S. P., Luo, G. \& Glumac, N. G. 2004 'Parametric effects on thin film growth and uniformity in an atmospheric pressure impinging jet CVD reactor.' J. Crystal Growth 267, 22-34.

${ }^{7}$ Kobayashi, R. \& Arai, T. 1990 'Spiral vortex behaviour in transition region and separation of three-dimensional boundary layers on spheres rotating in axial flow.' In Laminar Turbulent Transition, IUTAM Symposium Toulouse, France, ed. D. Arnal \& R. Michel, 551-557.

${ }^{8}$ Kobayashi, R. \& Izumi, H. 1983 'Boundary-layer transition on a rotating cone in still fluid.' J. Fluid Mech. 127, 353-64.

${ }^{9}$ Kobayashi, R., Kohama, Y. \& Kurosawa, M. 1983 'Boundary-layer transition on a rotating cone in axial flow.' J. Fluid Mech. 127, 341-52.

${ }^{10}$ Конама, Y. 1984 'Behaviour of spiral vortices on a rotating cone in axial flow.' Acta Mech. 51, 105-17.

${ }^{11}$ Kohama, Y. 1985 'Flow structures formed by axisymmetric spinning bodies.' AIAA J. 23, 1445.

${ }^{12}$ Kонама, Y. 1985 'Turbulent transition process of the spiral vortices appearing in the laminar boundary layer of a rotating cone.' Phys.-Chem. Hydrodyn. 6(5), 659.

${ }^{13}$ Kobayashi, R. 1994 'Review: Laminar-to-Turbulent Transition of Three-Dimensional Boundary Layers on Rotating Bodies.' Trans. ASME 116, 200-11.

${ }^{14}$ TAniguchi, H., Kobayashi, R. \& FUKUnishi, Y. 1998 'Stability of the boundary layer on a sphere rotating in still fluid.' Acta Mech. 129, 243-253.

${ }^{15}$ KobaYASHI, R. 1981 'Linear stability theory of boundary layer along a cone rotating in axial flow.' Bull. Japan Soc. Mech. Engrs. 24, 934-940.

${ }^{16}$ Kobayashi, R., Kohama, Y. \& Kurosawa, M. 1983 'Boundary-layer transition on a rotating cone in axial flow.' J. Fluid Mech. 127, 341-352.

${ }^{17}$ Salzberg, F. \& Kezios, S. P. 1965 'Mass transfer from a rotating cone in axisymmetric flow.' J. Heat Transfer 87 , $469-476$. 
${ }^{18}$ Garrett, S. J. 2002 'The stability and transition of the boundary layer on rotating bodies.' PhD thesis, Cambridge University.

${ }^{19}$ Garrett, S. J. \& Peake, N. 2002 'The stability and transition of the boundary layer on a rotating sphere.' J. Fluid Mech. 456, 199-217.

${ }^{20}$ Garrett, S. J. \& Peake, N. 2004 'The stability of the boundary layer on a rotating sphere in a uniform axial flow.' European J. Mech., B., 23, 241-253.

${ }^{21}$ Garrett, S. J. \& Peake, N. 2007 'The absolute instability of the boundary layer on a rotating cone.' European. J. Mech. B. 26, 344-53.

${ }^{22}$ Garrett, S. J. \& Peake, N. 2007 'On the global linear stability of the boundary layer on rotating bodies.' Advances in Turbulence XI: Proceedings of the 11th EUROMECH European Turbulence Conference, ed. J.M.L.M Palma \& A. Silva Lopes, $550-552$.

${ }^{23}$ Garrett, S. J., Hussain, Z. \& Stephen, S. O. 2009 'The crossflow instability of the boundary layer on a rotating cone.' J. Fluid Mech. 622, 209-232.

${ }^{24}$ Hussain, Z., Garrett, S. J. \& Stephen, S. O. 2009 'The convective instability of the boundary layer on a rotating disk in axial flow.' Submitted.

${ }^{25}$ Lingwood, R. J. 1995 'Absolute instability of the boundary layer on a rotating disk.' J. Fluid Mech. 299, 17-33.

${ }^{26}$ LingwoOD, R. J. 1996 'An experimental study of absolute instability of the rotating-disk boundary layer flow.' J. Fluid Mech. 314, 373-405.

${ }^{27}$ Rosenhead, L. 1963 Laminar Boundary Layers. Oxford.

${ }^{28}$ Evans, H. 1968 Laminar Boundary Layer Theory. Addison-Wesley.

${ }^{29} \mathrm{KoH}$, J. C. Y. \& Price, J. F. (1967) 'Non-similar boundary-layer heat transfer on a rotating cone in axial flow.' J. Heat Transfer 89, 139-145.

${ }^{30}$ Corke, T. C. \& KNAsiak, K. F. 1998 'Stationary travelling cross-flow mode interactions on a rotating disk' J. Fluid Mech. 355, 285-315.

${ }^{31}$ MALIK, M. R. 1986 The neutral curve for stationary disturbances in rotating-disk flow. J. Fluid Mech. 164, 275-87.

${ }^{32}$ Davies, C. \& CARpenter, P. W. 2003 'Global behaviour corresponding to the absolute instability of the rotating-disk boundary layer.' J. Fluid Mech. 486, 287-329.

${ }^{33}$ Pier, B., Huerre, P. \& Chomaz, J.-M. 2001 Bifurcation to fully nonlinear synchronized structures in slowly varying media. Physica D. 148, 49-93.

${ }^{34}$ Pier, B. \& Huerre, P. 2001 Nonlinear self-sustained structures and fronts in spatially developing wake flows. J. Fluid Mech. 435, 359-381.

${ }^{35}$ PIER, P. 2002 Fully nonlinear waves and transition in the boundary layer over a rotating disk: Advances in Turbulence IX, Proceedings of the 9th European Turbulence Conference, ed by I. P. Castro \& P. E. Hancock. 